\title{
Análise da equidade na distribuição do financiamento federal à Atenção Primária à Saúde entre os municípios do Ceará
}

Analysis of equity in the distribution of federal funding to primary health care among the municipalities of Ceará, Brazil

Análisis de equidad en la distribución del financiamiento federal a la atención primaria de salud entre los municipios de Ceará, Brasil

Natália Lima Sousa ${ }^{1}$

Maria Helena Lima Sousa ${ }^{2}$

Marcelo Gurgel Carlos da Silva ${ }^{3}$

\section{Resumo}

Objetivo: analisar a equidade na distribuição dos recursos da Atenção Primária à Saúde nos municípios do estado do Ceará. Metodologia: trata-se de um estudo ecológico, utilizando dados secundários de domínio público dos 184 municípios cearenses, entre 2004 e 2014. Coletou-se dados populacionais no Departamento de Informática do Sistema Único de Saúde; recursos recebidos de custeio da atenção primária no Fundo Nacional de Saúde e índice de desenvolvimento municipal no Instituto de Pesquisa e Estratégia Econômica do Ceará. Calculou-se o gasto com atenção básica total e per capita por porte populacional dos municípios, sendo a análise realizada por gráficos de dispersão e boxplot. Resultados: foi revelada variação de aumento sequencial entre municípios de grande porte>municípios de pequeno porte>municípios de médio porte. O gasto per capita com a atenção básica apresentou variação: municípios de médio porte>municípios de grande porte>municípios de pequeno porte. Entre 2000 e 2014, o gasto com atenção básica per capita sobressai nos municípios de pequeno porte, passando de $R \$ 45,23$ (2004) para $R \$ 132,97$ (2014); nos municípios de médio porte de $R \$ 32,52$ para $R \$ 97,05$; e municípios de grande porte, de $R \$$ 29,14 para $\mathrm{R} \$ 82,10$. Análises gráficas constataram maior crescimento dos gastos entre municípios de pequeno porte, de 2004 e 2012, apresentando queda em 2014. Conclusão: a política de financiamento da atenção primária do governo federal beneficiou os municípios de pequeno porte, apresentando tendência crescente de recursos entre 2000 e 2012, porém, em 2014, essa tendência se inverteu, o que é preocupante para a manutenção da Atenção Primária como base do Sistema Único de Saúde.

\section{Palavras-chave}

Equidade. Financiamento. Atenção Primária à Saúde.

\footnotetext{
Abstract

Objective: to analyze the equity in the distribution of resources of primary health care in municipalities of the state of Ceará. Methods: this is an ecological study, using secondary data from the public domain of 184 municipalities in Ceará, between 2004 and 2014.

${ }^{1}$ Mestre em Saúde Coletiva, Universidade Estadual do Ceará, Fortaleza, Ceará, Brasil. https://orcid.org/0000-0001-70706138. E-mail: natalia.Is1@hotmail.com

2 Doutora em Saúde Coletiva, Universidade Estadual do Ceará, Fortaleza, Ceará, Brasil; professora visitante, Programa de Pós-Graduação em Saúde Coletiva e Mestrado profissional em Gestão da Saúde, Universidade Estadual do Ceará, Fortaleza, Ceará, Brasil. https://orcid.org/0000-0002-4134-2483. E-mail: helena.Is68@gmail.com

${ }^{3}$ Pós-doutor, Economia da Saúde, Universidade de Barcelona, Barcelona, Espanha; professor titular, Programa de Pósgraduação em Saúde Coletiva, Universidade Estadual do Ceará, Fortaleza, Ceará, Brasil. https://orcid.org/0000-0003-40301206. E-mail: marcelo.gurgel@uece.br
} 
Population data were collected at the Informatics Department of the Unified Health System; resources were received from the cost of primary care in the National Health Fund and municipal development index in the Institute of Research and Economic Strategy of Ceará. The expenditure on total primary care and per capita was calculated by population size of the municipalities, and the analysis was performed using scatterplots and boxplots. Results: a sequential increase variation was revealed between large municipalities $>$ small municipalities> medium-sized municipalities. Per capita spending on primary care are varied: medium-sized municipalities > large municipalities > small-sized municipalities. Between 2000 and 2014 , spending on primary health care per capita stands out in small municipalities, from R \$ 45.23 (2004) to R \$ 132.97 (2014); in medium-sized municipalities from R \$ 32.52 to R \$ 97.05; and large municipalities, from $R$ \$ 29.14 to $R$ \$ 82.10. Graphical analyzes found greater growth in spending between small municipalities, from 2004 to 2012, decreasing in 2014. Conclusion: the federal government's primary health care financing policy benefited small municipalities, with an increasing trend of resources between 2000 and 2000. 2012, however, in 2014, this trend was reversed, which is worrying for the maintenance of Primary Care as the basis of the Unified Health System.

\section{Keywords}

Equity. Financing. Primary health care.

\section{Resumen}

Objetivo: analizar la equidad en la distribución de recursos de Atención Primaria de Salud en los municipios del estado de Ceará. Metodología: se trata de un estudio ecológico, utilizando datos secundarios de dominio público de 184 municipios de Ceará, entre 2004 y 2014. Los datos poblacionales fueron recolectados en el Departamento de Informática del Sistema Único de Salud; recursos recibidos del costo de atención primaria en el Fondo Nacional de Salud e índice de desarrollo municipal en el Instituto de Investigación y Estrategia Económica de Ceará. El gasto en atención primaria total y per cápita se calculó por tamaño de población de los municipios, y el análisis se realizó mediante diagramas de dispersión y diagramas de caja. Resultados: se reveló una variación de aumento secuencial entre municipios grandes> municipios pequeños> municipios medianos. El gasto per cápita en atención primaria varió: municipios medianos $>$ municipios grandes $>$ municipios pequeños. Entre 2000 y 2014, se destaca el gasto en atención primaria de salud per cápita en los municipios pequeños, de R \$45,23 (2004) a R \$132,97 (2014); en municipios medianos de $\mathrm{R} \$ 32,52$ a $\mathrm{R} \$ 97,05$; y grandes municipios, de $\mathrm{R} \$ 29,14$ a $\mathrm{R} \$ 82,10$. Los análisis gráficos encontraron un mayor crecimiento en el gasto entre municipios pequeños, de 2004 a 2012, disminuyendo en 2014. Conclusión: la política de financiamiento de la atención primaria de salud del gobierno federal benefició a los municipios pequeños, con una tendencia creciente de recursos entre 2000 y 2000. 2012, sin embargo, en En 2014 se revirtió esta tendencia, preocupante para el mantenimiento de la Atención Primaria como base del Sistema Único de Salud.

\section{Palabras clave}

Equidad. Financiación. Primeros auxilios.

\section{Introdução}

A Atenção Primária à Saúde (APS) é a porta de entrada do sistema público de saúde no Brasil, ou seja, considera o sujeito em sua singularidade, complexidade, integralidade e inserção sociocultural e busca promoção de sua saúde, a prevenção e tratamento de 
doenças e a redução de danos ou de sofrimentos que possam comprometer suas possibilidades de viver modo saudável (1).

A universalização da saúde, por meio do Sistema Único de Saúde (SUS), trouxe consigo uma gama de desafios para a gestão pública. O seu financiamento constitui-se um dos pontos cruciais do debate na busca de se atingir o objetivo de dotar os entes federados de mecanismos que possam entrar em sintonia com a política oficial da saúde no País sem comprometer as diretrizes propostas pelo Ministério da Saúde (2).

Em 1994, inicia-se uma nova formatação da Atenção Básica da Saúde pelo SUS, incorporando-se a estruturação do Programa Saúde da Família (PSF) como alternativa ao modelo tradicional, baseado na doença, com atuação exclusiva sobre a demanda espontânea (4).

Em dezembro de 2018, o Ceará possuía nove milhões de habitantes e 2.403 equipes de PSF, o que correspondia a uma cobertura de 7,2 milhões de habitantes, ou 79,84\% do contingente populacional. Segundo estimativas do Ministério da Saúde, a atenção básica no Ceará deveria abranger 7,3 milhões de habitantes, o que corresponderia a um percentual de $81,96 \%$ da população (5).

Em 1998, de acordo com a Portaria $n^{\circ} 3.925$, o governo federal aprovou o Manual para Organização da Atenção Básica no Sistema Único de Saúde e cria os recursos do piso da atenção básica (PAB), repassados pelo Fundo Nacional de Saúde (FNS) diretamente aos Fundos Municipais de Saúde, com a recomendação para que os recursos estejam identificados nos orçamentos municipais como receita de transferências intergovernamentais provenientes da esfera federal, vinculada diretamente ao Fundo Municipal de Saúde (6).

Segundo o Conselho Nacional de Secretários de Saúde (1), além do PAB fixo, foi definido também um PAB variável, baseado em incentivos para a implementação do PSF, dentre outros, respeitando as peculiaridades regionais, o que elevou significativamente os recursos nesse nível de atenção. Entre 1995 e 2004, o gasto com atenção primária ganhou importância relativa e absoluta no total de despesas com ações e serviços públicos de saúde (ASPS). Em 1996, representavam 10,82\% da despesa com ASPS. Em 2004, a participação subiu para 18,34\%, ou seja, passou de $R$ \$ 2.879 milhões em 1995 para $R$ \$ 3.409 milhões em 2004 (7).

Esse acréscimo ocorreu graças à vigência da Emenda Constitucional nº 29/2000 (8), que definiu uma política de financiamento da saúde para a União, Estados, Municípios e 
Distrito Federal, fixando, a partir de $2004,12 \%$ da receita vinculada para os Estados e Distrito Federal, 15\% para os Municípios e, para a União, o valor empenhado no ano anterior corrigido pela variação nominal do Produto Interno Bruto (PIB).

Em 2006, o governo federal redefiniu a Política Nacional da Atenção Básica (PNAB), que incluiu a desfragmentação do financiamento da $A B S$ e determinou que o somatório da parte fixa e variável do PAB comporá o teto financeiro do bloco atenção básica, conforme estabelecido nas diretrizes do Pacto pela Vida em Defesa do SUS e da Gestão (8).

Giovanella (9) pontua que o sucesso da expansão da atenção básica do SUS no país e das mudanças alcançadas, ainda que parciais, de modelo assistencial (com generalista, equipe multiprofissional, agentes comunitários de saúde, orientação comunitária, territorialização) decorrem da continuidade da indução financeira do modelo Estratégia Saúde da Família (ESF) sustentada ao longo do tempo.

A APS é um componente extremamente relevante para destinação de recursos financeiros, pois, por mais de vinte anos, tem sido reconhecida como um dos componenteschave de um sistema de saúde eficaz (10).

Para a Organização Pan Americana de Saúde (OPAS), um sistema de saúde com base na APS requer uma estratégia abrangente para a organização e operacionalização dos sistemas de saúde e que considere o direito ao maior nível possível de saúde - sua principal meta -, maximizando a equidade e a solidariedade (10).

O conceito de equidade expresso na nova portaria da Política Nacional da Atenção Básica (2017) é entendido como a oferta do cuidado, reconhecendo as diferenças nas condições de vida e de saúde e de acordo com as necessidades das pessoas, considerando que o direito à saúde passa pelas diferenciações sociais e deve atender à diversidade. Portanto, proíbe qualquer exclusão baseada em idade, gênero, cor, crença, nacionalidade, etnia, orientação sexual, identidade de gênero, estado de saúde, condição socioeconômica, escolaridade ou limitação física, intelectual, funcional, entre outras, com estratégias que permitam minimizar desigualdades, evitar exclusão social de grupos que possam vir a sofrer estigmatização ou discriminação, de modo a impactar na autonomia e na situação de saúde (11).

Equidade tem sido, portanto, uma expressão bastante utilizada quando tratamos de direitos sociais, tanto em sentido mais genérico quanto em um mais específico. No primeiro, termina por confundir-se com o próprio sentido de justiça, na perspectiva do estabelecimento 
de regras justas para a vida em sociedade, o que a aproxima do conceito e do sentido de igualdade, frequentemente assim entendidos no âmbito da saúde (12-14).

Para efeito deste estudo, o conceito de equidade corresponde ao princípio segundo o qual a distribuição de recursos é feita em função das necessidades de saúde de uma determinada população.

Com base na evolução e na política nacional de financiamento da APS nos municípios cearenses a questão norteadora deste estudo é: houve redução das desigualdades entre municípios cearenses no financiamento da APS, no período de 2004 a 2014? Considerouse que, nesse período, encontravam-se a vigência da Contribuição Provisória sobre Movimentação Financeira (CPMF) de 1997 a 2007, a edição da Emenda Constitucional n. ${ }^{\circ}$ 29/2000, a Emenda Complementar n 141/2012 e as Portarias GM/MS n 1.409/2013, dentre outras.

Ressalta-se que são raros os estudos relacionados à equidade no financiamento público na APS nas bases de dados on-line, o que limita a ação de planejamento e orçamento dos recursos para a saúde.

Nesse sentido, o objetivo deste estudo é analisar a equidade no financiamento federal na Atenção Primária à Saúde entre os municípios do estado do Ceará.

\section{Metodologia}

Esta pesquisa caracteriza-se como um estudo ecológico que são estudos que abordam áreas geográficas, analisando comparativamente indicadores globais, quase sempre por meio de correlação entre variáveis ambientais (ou socioeconômicas) e indicadores de saúde. Nesse estudo, a área ecológica pode sintetizar um conjunto enorme de variáveis, aproximando mais esse tipo de estudo da realidade social concreta (15).

Foram utilizados dados secundários de domínio público referentes ao financiamento federal da Atenção Primária à Saúde (APS) nos 184 municípios que fazem parte do estado do Ceará, localizado na região Nordeste do país. Segundo o Instituto Brasileiro de Geografia e Estatística (IBGE) (16), a população estimada, em 2019, é de 9,1 milhões, com crescimento populacional de 0,6\% entre 2018 e 2019.

O período de estudo são os anos pares entre 2004 e 2014. A escolha dos anos pares deve-se à disponibilidade de informações sobre o Índice de Desenvolvimento Municipal (IDM) pelo Instituto de Pesquisa e Estratégia Econômica do Ceará (IPECE). 
As informações utilizadas no estudo foram: a) recursos recebidos para o custeio do piso de atenção básica da saúde (PAB), composto do PAB fixo e do PAB fixo ampliado; b) o piso de atenção básica da saúde variável (PAB-variável), composto dos seguintes incentivos: estratégia saúde da família; saúde bucal; assistência financeira complementar (AFC); agente comunitário de saúde (ACS); serviço de atenção domiciliar (SAD); custeio para os Núcleos de Apoio à Saúde da Família (NASF); Programa Academia da Saúde (custeio); financiamento do Programa Nacional de Melhoria do Acesso e da Qualidade da Atenção Básica; equipes do consultório na rua (custeio); Programa Nacional de Melhorias do Acesso e Qualidade da Atenção Básica; ESF de municípios e de profissionais integrantes do PROVAB e Mais Médicos e Programa Saúde da Família (PSF); e o IDM dos municípios.

Segundo o IPECE (17), o IDM carrega em sua essência a ideia de uma análise multidimensional, que por meio de técnicas estatísticas, traduz o nível de desenvolvimento relativo de cada um dos municípios cearenses em um indicador sintético. Ao todo, são trabalhados 30 indicadores agrupados em quatro grupos ligados aos aspectos fisiográficos, fundiários e agrícolas; demográficos e econômicos; de infraestrutura de apoio; e sociais.

O modelo estatístico é desenvolvido para cada grupo de indicadores, cujo índice é calculado a partir da aplicação de técnicas multivariadas de análise fatorial e do método de componentes principais. Para classificar os municípios segundo o IDM, bem como os índices obtidos em cada grupo específico, utilizou-se a análise de agrupamento (cluster analysis) com o método dek-médias para as partições das classes. Esse método é um dos mais usados em análise de agrupamentos quando se tem muitos elementos.

Em seguida, calculou-se o PAB total (PAB fixo + PAB variável) e o PAB per capita (PAB total $/ n .^{\circ}$ de habitantes) por município e por porte populacional.

Esses dados foram coletados nas seguintes fontes: transferências federais para a atenção primária dos municípios no Fundo Nacional de Saúde (FNS); dados populacionais no site do Departamento de Informática do SUS (DATASUS); e o Índice de Desenvolvimento Municipal (IDM) no Instituto de Pesquisa e Estratégia Econômica do Ceará (IPECE).

Para análise dos resultados, utilizou-se como referência o porte dos municípios utilizado por Sousa (18), que são: municípios de pequeno porte (população < 30 mil habitantes); municípios de médio porte (30 mil hab. $\leq$ população < 100 mil hab.); e municípios de grande porte (população $\geq 100$ mil hab.).

Para análise dos dados, primeiramente se fez uma panorâmica do financiamento da APS no estado, no período considerado para o estudo. Depois, relacionou-se os dados de 
financiamento federal da ABS com o IDM por meio dos quadros de medidas descritivas, com objetivo de observar o crescimento dos valores médios para os indicadores, bem como a porcentagem do crescimento do financiamento.

Foram também utilizados gráfico de dispersão para avaliar a forma de distribuição desses indicadores; gráfico de dispersão para avaliar a relação e a distribuição anual e/ou por porte dos municípios do piso de atenção básica (PAB) total e do PAB per capita, em função do IDM; e o gráfico boxplot relacionando o PAB per capita por porte de municípios.

Para elaboração dos gráficos, os dados foram organizados no aplicativo Excel e transportados para o aplicativo SPSS, versão 19.

Os dados monetários foram corrigidos pelo Índice Geral de Preços de Mercado (IGPM), tendo por ano base 2014.

\section{Resultados e discussão}

O financiamento federal do PAB total apresentou aumento real em todos os portes de município, entretanto, em termos de valor absoluto, houve maior aporte de recursos nos municípios de grande porte (MGPs), seguido dos municípios de médio porte (MMPs) e, por fim, dos municípios de pequeno porte (MPPs). Na sequência, os valores variaram percentualmente em $255,8 \%, 215,4 \%$ e $202,5 \%$ (Tabela 1 ).

Quando se observa o valor per capita, verifica-se, todavia, que o maior acréscimo de recursos por população assistida beneficiou primeiramente os MMPs, seguido dos MGPs e, por fim, dos MPPs.

Em termos de valores per capita, os MPPs foram mais beneficiados com as políticas de financiamento federal para a APS porque os gastos per capita apresentaram uma tendência crescente, ou seja, os MPPs passaram de $R \$ 45,23$ em 2004 para $R \$ 132,97$ em 2012, sofrendo uma queda de R\$ 6,9 por pessoa entre 2012 e 2014. Os recursos disponíveis, que estavam subindo $29 \%$ em média, tendo chegado à faixa de aumento de $37,3 \%$ entre 2010 e 2012, apresentaram uma queda de 7\% entre 2012 e 2014. 
Tabela 1. Recursos de custeio financiados pelo Governo Federal para a Atenção Primária à Saúde (APS) para municípios de grande porte (MGPs), médio porte (MMPs) e pequeno porte (MPPs) nos anos pares de 2004 a 2014

\begin{tabular}{c|c|c|c|c|c|c}
\hline \multirow{2}{*}{ Ano/Porte } & \multicolumn{2}{|c|}{ MGP } & \multicolumn{2}{c|}{ MMP } & \multicolumn{2}{c}{ MPP } \\
\cline { 2 - 7 } & $\begin{array}{c}\text { PAB total } \\
(\mathrm{R} \$ 1,00)\end{array}$ & $\begin{array}{c}\mathrm{PAB} \text { per capita } \\
(\mathrm{R} \$)\end{array}$ & $\begin{array}{c}\text { PAB total } \\
(\mathrm{R} \$ 1,00)\end{array}$ & $\begin{array}{c}\mathrm{PAB} \text { per capita } \\
(\mathrm{R} \$)\end{array}$ & $\begin{array}{c}\text { PAB total } \\
(\mathrm{R} \$ 1,00)\end{array}$ & $\begin{array}{c}\text { PAB per } \\
\text { capita }(\mathrm{R} \$)\end{array}$ \\
\hline $\mathbf{2 0 0 4}$ & 69.784 .772 & 29,14 & 84.711 .680 & 32,53 & 79.035 .966 & 45,23 \\
$\mathbf{2 0 0 6}$ & 94.024 .082 & 33,01 & 98.994 .773 & 38,64 & 111.718 .898 & 58,27 \\
$\mathbf{2 0 0 8}$ & 125.634 .606 & 44,68 & 142.683 .144 & 53,01 & 143.109 .601 & 73,68 \\
$\mathbf{2 0 1 0}$ & 162.996 .193 & 54,06 & 178.598 .912 & 66,79 & 186.543 .593 & 96,88 \\
$\mathbf{2 0 1 2}$ & 224.381 .927 & 73,96 & 261.418 .533 & 95,08 & 252.897 .593 & 132,97 \\
$\mathbf{2 0 1 4}$ & $\mathbf{2 4 8 . 3 0 8 . 1 2 8}$ & 82,10 & $\mathbf{2 6 7 . 1 4 3 . 9 6 9}$ & 97,05 & 239.058 .433 & 126,02 \\
\hline $\mathbf{V}$ Var \% & $\mathbf{2 5 5 , 8}$ & $\mathbf{1 8 1 , 7 8}$ & $\mathbf{2 1 5 , 4}$ & $\mathbf{1 9 8 , 3}$ & $\mathbf{2 0 2 , 5}$ & $\mathbf{1 7 8 , 6}$ \\
$\mathbf{2 0 0 4 / 2 0 1 4}$ & & &
\end{tabular}

Fonte: elaboração própria, a partir de dados da FNS, DATASUS/MS e IPECE.

Segundo Barros (19), o gráfico de dispersão é um tipo de representação bastante útil quando há interesse em explorar o comportamento de duas variáveis simultaneamente, uma em relação a outra.

Pelos resultados do gráfico 1, percebe-se que, no ano de 2004, os municípios distribuídos por porte populacional estão bastante adstritos, crescendo gradativamente, mas em ritmos diferentes. A relação entre o IDM e o PAB per capita foi mais expressiva nos MPPs, seguida dos MMPs e, por último, dos MGPs. Em todo o período estudado, vê-se que nos MPPs houve melhor distribuição de recursos, atingindo no máximo 67,4\% (2004) e, no mínimo, 64,7\% (2014). Nos MMPs, observou-se o máximo de 30,4\% e o mínimo de 28,8\%. Entre os MGPs teve o máximo de 4,9\% e o mínimo de 3,8\% (Gráfico 1).

Em todos os portes de municípios, vê-se que houve uma melhor distribuição de recursos, em especial os MPPs (Gráfico 1).

Nos três grupos, a partir dos anos de 2010, nota-se um crescimento relacionado ao valor por habitante. Pode-se dizer que alguns municípios, representados por pontos mais dispersos, estão recebendo mais recursos do PAB per capita (Gráfico 1). 
Gráfico 1. Relação do PAB per capita e o Índice de Desenvolvimento Municipal (IDM) nos anos pares de 2004 a 2014, apresentado pelo gráfico de dispersão de acordo com o porte do município

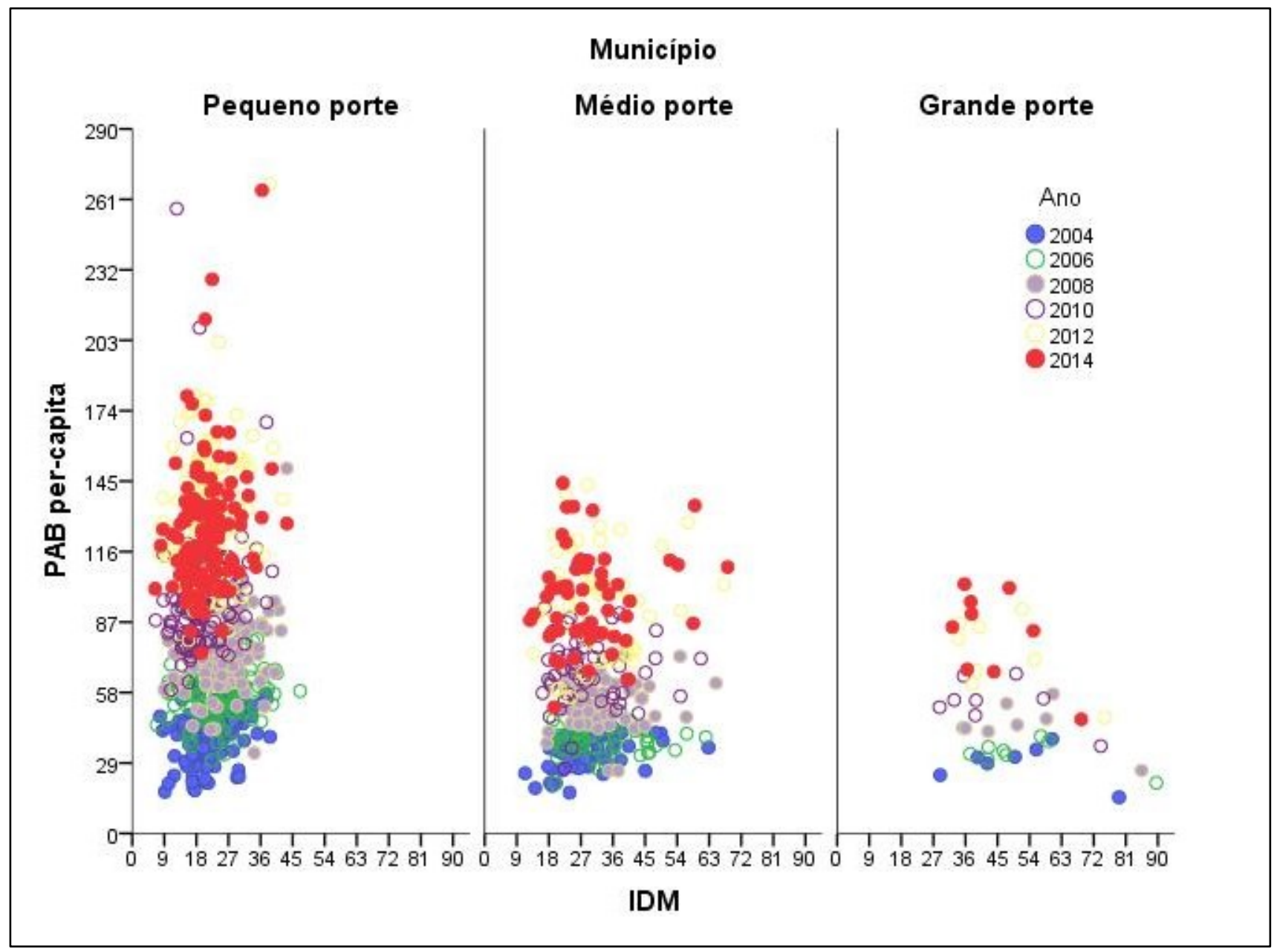

Fonte: elaboração própria.

O gráfico 2 apresenta a relação entre o PAB per capita e o IDM estratificado por ano de estudo nos municípios de grande, médio e pequeno portes. É interessante perceber o crescimento da relação entre o PAB per capita e o IDM até o ano de 2012, havendo uma pequena redução entre os MPPs no ano de 2014. 
Gráfico 2. Relação do piso de atenção básica (PAB) per capita e o Índice de Desenvolvimento Municipal (IDM) estratificado por anos pares de 2004 a 2014

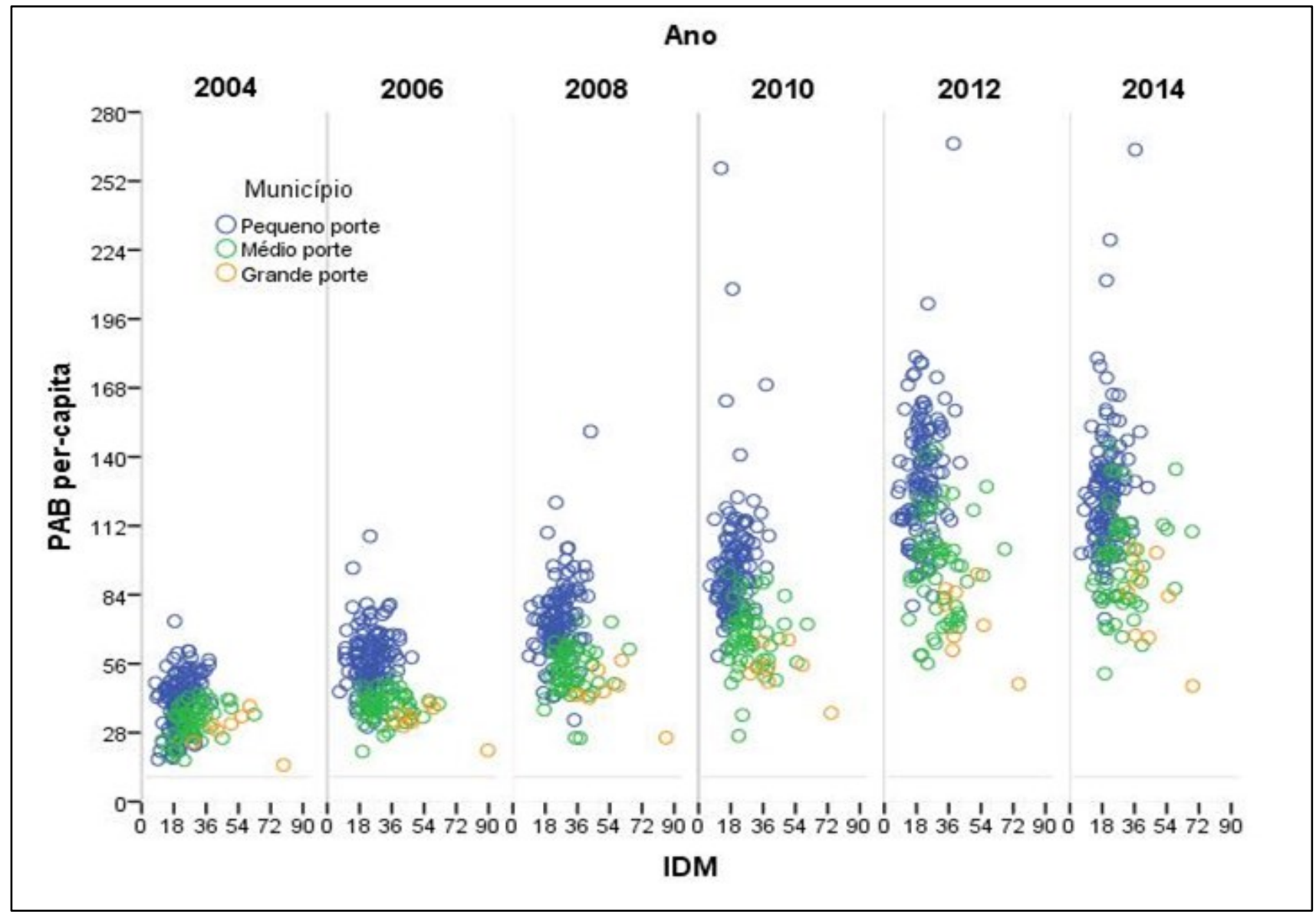

Fonte: elaboração própria.

O gráfico 3 é uma representação do mesmo fenômeno dos gráficos 1 e 2, pelo gráfico de histograma, o qual é similar a um gráfico de colunas, mas não devem existir espaços entre as colunas porque se trata de um tipo apropriado à representação de variáveis quantitativas. A largura da coluna representa o intervalo de valores da variável, enquanto a altura de cada coluna corresponde à frequência de dados observados dentro do respectivo intervalo de classe. Nesse gráfico, observa-se a evolução do crescimento do PAB per capita em relação ao IDM, iniciando o ano de 2004 com a formação mais desigual dessa relação, cujo crescimento é verticalizado e mais concentrado. Nos anos subsequentes, o gráfico se horizontaliza, o que mostra uma melhor distribuição dos recursos entre municípios de menor nível socioeconômico. 
Gráfico 3. Relação do piso de atenção básica (PAB) per capita e o Índice de Desenvolvimento Municipal (IDM) apresentado por anos pares de 2004 a 2014

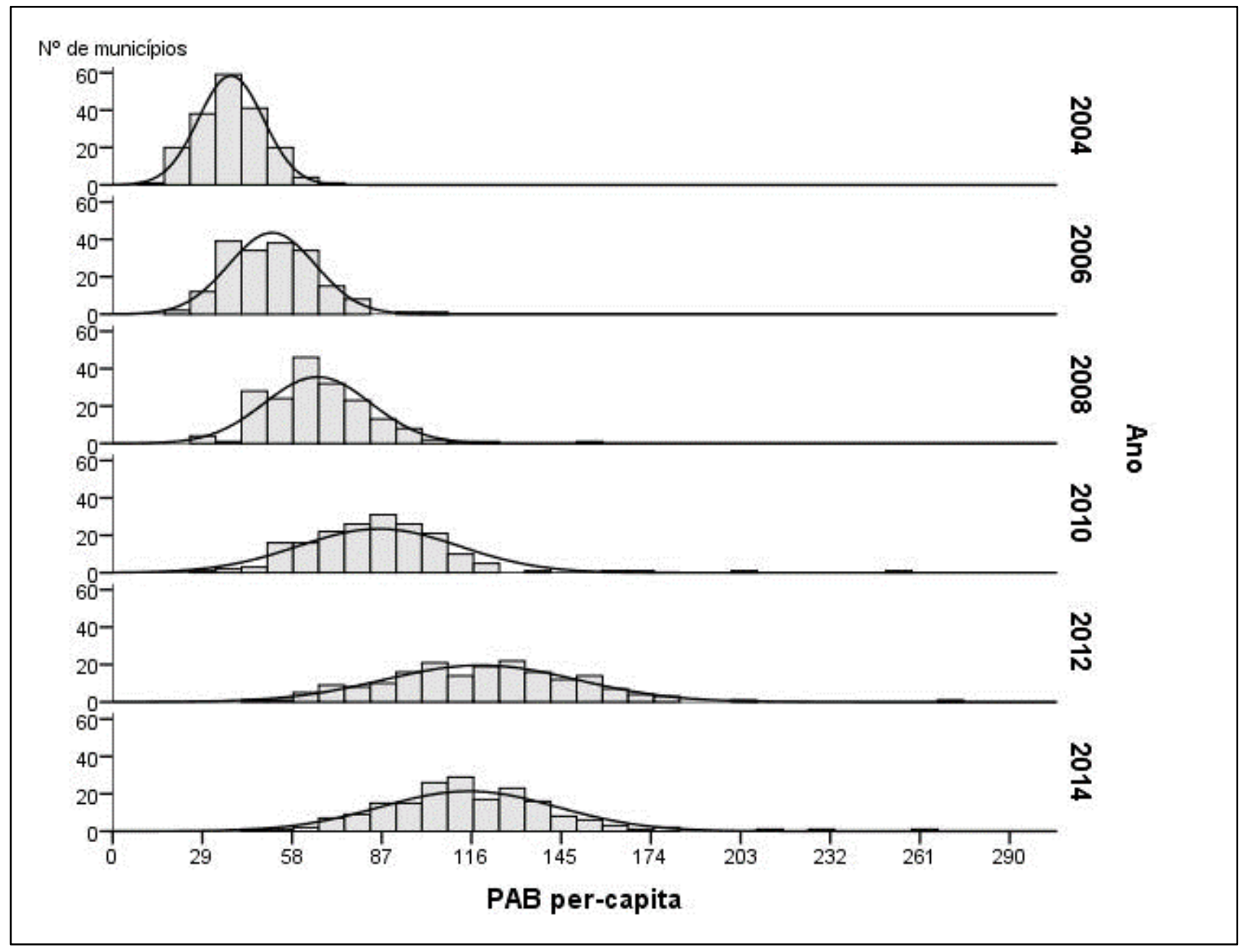

Fonte: elaboração própria.

Apesar de variações, o gráfico 3 é um retrato positivo da distribuição no PAB per capita e do IDM entre os anos de 2004 a 2012. O motivo desse resultado decorre da Emenda Constitucional n² 29/2000; da Lei Complementar n 141/2012; da evolução das pactuações no financiamento da saúde, como, por exemplo, o Pacto pela Saúde em 2006; as transferências dos recursos federais através de blocos de financiamento em 2007; e da Portaria Nacional de Atenção Básica, em 2012.

Contextualizando um pouco o histórico do financiamento, o Pacto pela Saúde veio para fortalecer os princípios constitucionais do SUS, dando ênfase às necessidades da população, e que implicará o exercício simultâneo de definição de prioridades articuladas e integradas nos três componentes: Pacto pela Vida, Pacto pela Defesa do SUS e o Pacto de Gestão do SUS (8). 
Os blocos de financiamento onde os repasses fundo a fundo se consolidaram permitiram um melhor monitoramento e controle. A Lei Complementar (LC) $n^{\circ} 141$, que dispõe os valores mínimos a serem aplicados anualmente pela União, estados e Distrito Federal em ações e serviços públicos de saúde, estabelece os critérios de rateio dos recursos de transferências para a saúde e as normas de fiscalização, avaliação e controle das despesas com saúde nas três esferas de governo. Segundo Andrade (20), o que alicerça a referida lei é a busca da garantia da equidade orçamentária regional, com a diminuição das disparidades regionais nos termos da Constituição Federal (CF).

Ressalta-se também que a PNAB (11) aborda todas as funções, diretrizes e princípios da atenção primária, suas responsabilidades em todas as esferas de governo, assim como o funcionamento, atribuições dos profissionais, as especificidades de cada estratégia (Estratégia Saúde da Família, Programa de Agentes Comunitários de Saúde, entre outros), além da educação permanente e tantos outros direcionamentos à APS.

Atualmente, uma preocupação é a reformulação da Nova Portaria da Atenção Básica, aprovada em setembro de 2017. Mesmo com amplo debate; embasamento teórico produzido pelas universidades e centros de pesquisas dedicados ao estudo da APS; vivência prática de inúmeros profissionais aplicados à saúde integral; e os robustos dados que comprovam a eficácia da ESF (21), o Ministério da Saúde ignorou essas informações, permanecendo com propostas que nos deixam inquietos quanto a possíveis e prováveis consequências.

O gráfico 4 é a representação da relação exibida pelos gráficos 2 e 3 de forma de boxplot. Para Barros (19):

[...] o gráfico boxplot - ou gráfico de caixas de bigodes - é um tipo de gráfico com a forma de um retângulo, no qual a borda inferior e a superior representam, respectivamente, o primeiro e o terceiro quartil (onde o eixo horizontal representa as variáveis) do conjunto de dados. A linha que atravessa o retângulo corresponde ao valor da mediana. A representação de dados por meio de um gráfico de caixas de bigodes é uma ferramenta poderosa para identificação de outlier (valores discrepantes) e para a análise da distribuição dos dados. (19)

Os MPPs (caixa azul) se destacam entre os anos de 2004 a 2012, mas em 2014, sofreu uma diminuição, o que pode sinalizar uma estagnação do processo de redução das desigualdades, podendo apresentar um aumento da iniquidade a partir desse momento. 
Gráfico 4. Relação do PAB per capita e o IDM apresentado por ano e porte do município, estratificado por anos pares de 2004 a 2014

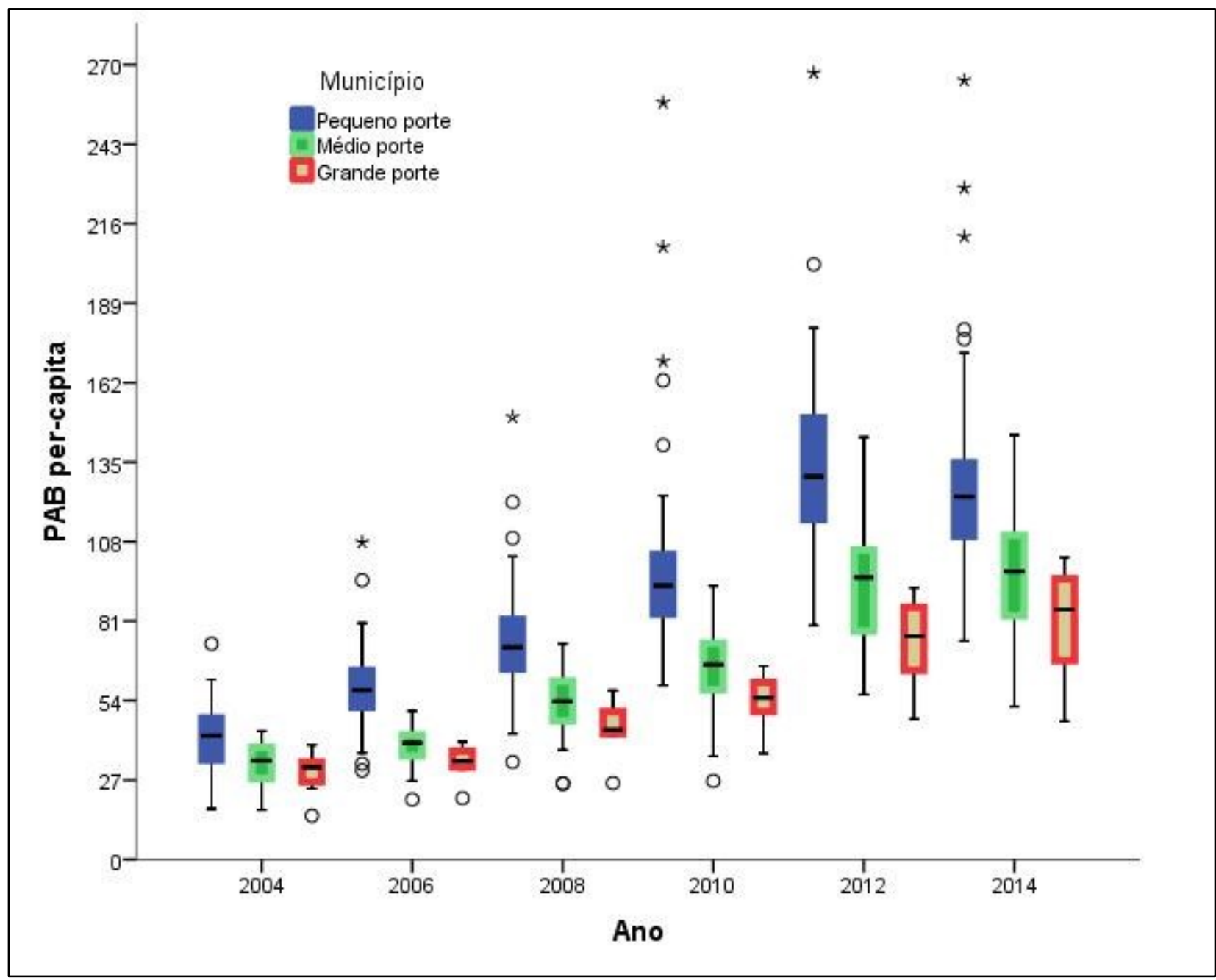

Fonte: elaboração própria.

Os MMPs, representados pelas caixas verdes, mostram uma tendência crescente, embora apresente uma pequena redução em 2014. O mesmo fenômeno acontece com os MGPs, que provavelmente atingiram melhor equidade, embora 2014 represente um freio na linha de tendência entre os anos de 2004 a 2012.

Uma preocupação se reflete nos tempos atuais, com o novo modelo de financiamento da APS pactuado em outubro de 2019 , que fragiliza ainda mais a PPS no SUS e potencializa o processo de desfinanciamento. Para Funcia (21), diante de tudo o que foi apresentado pelo Ministério da Saúde (MS), abre-se o seguinte pressuposto: como será garantida a linha de cuidados em saúde baseada nos princípios e diretrizes constitucionais da universalidade, integralidade e equidade com a mudança de critérios de rateio para a transferência de recursos do Fundo Nacional de Saúde para os Fundos Estaduais e Municipais de Saúde 
para as ações de atenção primária, com a ênfase no cadastramento dos usuários, na produtividade e do desempenho das equipes?

\section{Conclusão}

Houve maior aporte de recursos totais entre os municípios de grande porte (MGPs), seguido dos municípios de médio porte (MMPs) e, por fim, dos municípios de pequeno porte (MPPs). Os MMPs se destacaram na variação percentual de aumento de recursos federais entre 2004 e 2014, sendo seguido pelos MGPs e, por último, os MPPs. Entretanto, em termos de valores do piso da atenção básica (PAB) per capita, os municípios de pequeno porte se sobressaem, apresentando valores superiores, seguidos dos municípios de médio porte e, por último, dos municípios de grande porte.

Apesar desse resultado em favor da redução das desigualdades no financiamento da Atenção Primária à Saúde (APS), o crescimento dos valores transferidos privilegiando os municípios de grande e médio porte pode reverter a tendência de distribuição equitativa de recursos beneficiando os MPPs, levando ao aumento das desigualdades em saúde entre os municípios de baixo nível socioeconômico.

Ressalta-se, como preocupação, a redução de recursos em 2014, possivelmente em consequência da crise econômica e política acontecida no país no início do ano, em razão da reeleição da presidente Dilma Rousseff, que culminou com seu impeachment em 2016.

É importante salientar que, mesmo havendo uma distribuição mais equitativa dos recursos federais destinados à APS, isso não significa dizer que esses recursos tenham sido suficientes para cobrir as necessidades de saúde da população cearense. Para tanto, necessitaria outro estudo mais aprofundado com esse objetivo.

Por fim, é importante registrar que, no decorrer de 2014 até o presente momento, temse como preocupação as medidas publicadas pelo governo federal que atingem o financiamento do Sistema Único e Saúde (SUS) e, em particular, da APS como a Emenda Constitucional (EC) $n^{\circ} 86 / 2015$, conhecida como Orçamento Impositivo e que estabelece a obrigatoriedade das emendas parlamentares, representando um valor expressivo dentro do orçamento do Ministério da Saúde; a EC n 87/2015, que aumentou de $20 \%$ para $30 \%$ a desvinculação dos recursos da União, quando não se sabe ao certo onde esses recursos serão aplicados; a EC n 95/2016, que congela os gastos públicos por 20 anos, atingindo sobremaneira as áreas sociais como saúde e educação; a Portaria n 2.979/2019, que modifica o financiamento do custeio da Atenção Primária à Saúde, de forma que a base de 
cálculo do PAB-fixo passa a ter como critério a população cadastrada, ao invés da população do município; e o novo financiamento da APS, pactuado em Comissão Intergestora Tripartite, em novembro de 2019, que vincula o financiamento a partir da quantidade de usuários cadastrados. Anteriormente, o financiamento se dava a partir da quantidade populacional do município.

A preocupação presente se situa no processo histórico de sub e desfinanciamento do SUS, o que será alargado após todos esses acontecimentos. Em uma população onde a grande maioria é SUS-dependente, essa realidade traz consequências diretas ao cotidiano das pessoas, além de confirmar a não aplicação dos deveres do Estado.

\section{Referências}

1. Conselho Nacional de Secretários de Saúde. O financiamento da saúde. Brasília: CONASS; 2011.

2. Brasil. Ministério da Saúde, Secretaria Executiva, Secretaria de Ciência, Tecnologia e Insumo Estratégico. Glossário temático: economia da saúde. $2^{a}$ ed. Brasília: MS; 2009.

3. Scatena JHG, Tanaka OY. Os instrumentos normalizadores (NOB) no processo de descentralização da saúde. Saúde e Sociedade. 2001;10(2):47-74.

4. Brasil. Ministério da Saúde. Saúde da Família: uma estratégia para a reordenação do Modelo Assistencial. Brasília: MS; 1997.

5. Brasil. Ministério da Saúde. Informações e Gestão da Atenção Básica [Internet]. Brasília: Ministério da Saúde; 2020 [citado em 5 out. 2019]. Disponível em https://egestorab.saude.gov.br/paginas/acessoPublico/relatorios/relHistoricoCoberturaAB.x html;jsessionid=bYoDBkPxzmAsWInOVmAYXFPf

6. Brasil. Ministério da Saúde. Portaria n 3.925, de 13 de novembro de 1988 [Internet]. Aprova o Manual para Organização da Atenção Básica no Sistema Único de Saúde, constante do Anexo I desta portaria. Brasília: Ministério da Saúde; 13 nov. 1988 [citado em 5 out. 2019]. Disponível em:

http://bvsms.saude.gov.br/bvs/saudelegis/gm/1998/prt3925_13_11_1998_rep.html

7. Chaui MS. Prefácio. In: Pochmann M. O mito da grande classe média: capitalismo e estrutura social. São Paulo: Boitempo; 2014. p.1-3.

8. Brasil. Conselho Nacional de Secretários de Saúde. Lei complementar $n^{\circ} 141$, de 13 de janeiro de 2012 [Internet]. Regulamenta o $\S 3^{\circ}$ do art. 198 da Constituição Federal para dispor sobre os valores mínimos a serem aplicados anualmente pela União, Estados, Distrito Federal e Municípios em ações e serviços públicos de saúde; estabelece os critérios de rateio dos recursos de transferências para a saúde e as normas de fiscalização, avaliação e controle das despesas com saúde nas 3 (três) esferas de governo; revoga 
dispositivos das Leis nos 8.080, de 19 de setembro de 1990, e 8.689, de 27 de julho de 1993; e dá outras providências. Brasília; 13 jan. 2012 [citado em 5 out. 2019]. Disponível em: http://www.planalto.gov.br/ccivil_03/leis/lcp/lcp141.htm

9. Giovanella L, Mendonça MHM. Atenção Primária à Saúde. In: Giovanella L. et al. Políticas e Sistema de Saúde no Brasil. 2 ed. Rio de Janeiro: Ed. Fiocruz; 2012. p. 12-25.

10. Organização Pan-americana da Saúde. Renovação da atenção primária em saúde nas Américas: documento de posicionamento da Organização Pan-americana da Saúde. Washington: OMS; 2007.

11. Brasil. Ministério da Saúde. Portaria $n^{\circ} 2.436$, de 21 de setembro de 2017 [Internet]. Aprova a Política Nacional de Atenção Básica, estabelecendo a revisão de diretrizes para a organização da Atenção Básica, no âmbito do Sistema Único de Saúde (SUS). Brasília; 21 set. 2017 [citado em 5 out. 2019]. Disponível em:

https://bvsms.saude.gov.br/bvs/saudelegis/gm/2017/prt2436_22_09_2017.html

12. Scatena JHG, Tanaka OY. Os instrumentos normalizadores (NOB) no processo de descentralização da saúde. Saúde e Sociedade. 2001;10(2):47-74.

13. Almeida LM, Rigolin TB. Geografia. 5 ed. São Paulo: Ed. Ática; 2002.

14. Campos GWS. Reflexões temáticas sobre equidade em saúde: o caso do SUS. Saúde e sociedade. São Paulo; 2006;15(2):23-33.

15. Barbosa LMM, Machado CB. Glossário de epidemiologia \& saúde. In: Rouquayrol MZ, Silva MGC, organizadores. Epidemiologia \& saúde. 8.ed. Rio de Janeiro: MedBook, 2018. 752p. ISBN 97885 8369-029-0.

16. Instituto Brasileiro de Geografia e Estatística. Cidades e Estados: Ceará. População estimada. [citado em 5 out. 2019]. Disponível em: https://www.ibge.gov.br/cidades-eestados/ce.html

17. Instituto de Pesquisa e Estratégia Econômica do Ceará. Ceará em números [Internet]. Fortaleza; 2016 [citado em 15 set. 2019]. Disponível em:

http://www2.ipece.ce.gov.br/publicacoes/ceara_em_numeros/2016/completa/Ceara_em_Nu meros_2016.pdf

18. Sousa MHL, Coelho VMC, Lima EC, Mota RMS, Fonseca MIF. Análise da equidade no financiamento federal na atenção básica da saúde no SUS-Ceará:

anos/2002/2004/2006/2008. In: Silva MGC, Sousa MHL. Temas de economia da saúde III: contribuições para a gestão do SUS. Fortaleza: Editora Eduece; 2011. p. 54-73.

19. Barros MVG, Reis RS, Hallal PC. Análise de dados em saúde. $3^{a}$ ed. Londrina: Midiograf; 2012.

20. Andrade LOM, Santos L, Ribeiro KG, Barreto ICHC. Política de saúde no Brasil: Epidemiologia e saúde. $8^{a}$ ed. Rio de Janeiro: Medbook; 2018. 
21. Funcia FR. Novo modelo de financiamento da Atenção Primária à Saúde pactuado na Comissão Intergestores Tripartite (CIT) em 31 de outubro de 2019: proposta de roteiro para reflexão, debates e demais providencias para o cumprimento da Constituição Federal, da legislação e das normas infralegais que regem o Sistema Único de Saúde [Internet]. Instituto de Direito Sanitário Aplicado, 1 nov. 2019 [citado em 5 out. 2019]. Disponível em: http://idisa.org.br/domingueira/domingueira-n-37-novembro-2019?lang=pt

\section{Colaboradores}

Sousa NL colaborou com a concepção; análise e interpretação de dados; redação; revisão crítica; e aprovação da versão final do artigo. Silva MGC e Sousa MHL contribuíram na revisão crítica e aprovação da versão final do artigo.

\section{Como citar este artigo}

Sousa NL, Silva MGC, Sousa MHL. Análise da equidade na distribuição do financiamento federal à Atenção Primária à Saúde entre os municípios do Ceará. Cadernos Ibero-Americanos de Direito Sanitário. 2021 jan./mar.;10(1):76-92. 